\title{
EVALUATION OF INTELLIGENCE SCORES AMONG STUDENTS FROM MOROCCAN URBAN AREAS
}

\author{
Khalid Marnoufi ${ }^{1}$, Bouzekri Touri ${ }^{1}$, Mohammed Bergadi ${ }^{1}, \&$ Imane Ghazlane ${ }^{1,2}$ \\ ${ }^{I}$ Multidisciplinary Laboratory of Information, Communication and Education Sciences and Technology \\ (LAPSTICE), Ben M'sik Faculty of Science, Hassan II University of Casablanca, post box 7955, \\ Casablanca (Morocco) \\ ${ }^{2}$ Higher Institute of Nursing and Health Technology Professions Casablanca-Settat, Morocco Street \\ Faidouzi Mohamed, 20250 Casablanca (Morocco)
}

\begin{abstract}
Our study is carried on Moroccan students from urban areas and will be the basis for calculating the full scale intelligence quotient FSIQ which positively predicts school results, for determining the psychometric profiles of the samples participating in this study, by administering the fifteen subtests of the Wechsler Intelligence Scale for Children and Adolescents - 5th Edition WISC V and for comparing the results to the average of the Wechsler tests. We have administered all subtests of WISC V, an individual general intelligence test for children aged between 6 and 16 based on the g-factor since the inception of the first Wechsler test. We ensure that every student participating in this study from the city of Safi has passed all the subtests. The participants are 101 students (59 girls and 42 boys) aged between 12 and 13 years and a few months. The results of the samples who are urban public school students show a median of full scale intelligence quotient, the average value of the verbal comprehension index is higher than the other four indexes of WISC V. In terms of correlations, the results show two types of relationships between the five indexes on the scale.
\end{abstract}

Keywords: FSIQ, WISC V, intelligence, students, urban.

\section{Introduction}

After a century of test development and theorizing, intelligence test scores remain a strong predictor of academic achievement (Deary, Strand, Smith, \& Fernandes, 2007; Rohde \& Thompson, 2007; Roth et al., 2015). The intelligence quotient estimates the development of intellectual capacity. However, many researchers have challenged the interpretation of the profiles with the intelligence quotient and have called for analysis at the level only (Glutting et al., 1997, 1988; McDermott et al., 1990; Watkins, 2000). The Wechsler Intelligence Scale for Children and Adolescents has emerged as a downward extension of an adult intelligence test. After the launch of the Wechsler Bellevue Intelligence Scale (WB), a rich history of research and clinical applications undoubtedly contributed to the development of WISC V. This Wechsler test is widely used for children from 6 years to 16 years and 9 months. It generally lasts between 65 and 80 minutes and produces a result reasonably typical of the standard measure of intelligence.

The developers are basing on the g-factor of Spearman, C. (1904). Thus, they stated that they were using the Cattell-Horn-Carroll Theory $(\mathrm{CHC})$, which is a synthesis of earlier theories on the hierarchical structure of human cognitive abilities. The first of these two theories is the Gf-Gc theory (Raymond Cattell, 1941; Horn, 1965), and the second is Carroll's hierarchical stratified mode (1993). The development of education in France is linked to a Binet Simon mental test; several tests over a century to date have been developed to solve school problems. Discovery-based learning researchers tend to focus their research on specific areas such as mathematics/numbers, computer skills, science, problem-solving and physical / motor skills (Alfieri, Brooks, Aldrich and Tenenbaum, 2011).

The objective of our study is to calculate and interpret the full-scale intelligence quotient FSIQ among Moroccan students in the urban environment using the fifth edition of the Wechsler Intelligence Scale for Children and Adolescents. It consists of 15 sub-tests Similarities, vocabulary, information, comprehension, block design, visual puzzles, matrix reasoning, figure weights, arithmetic, digit span, picture span, letter-number sequencing, coding, symbol search and cancellation. The full scale intelligence quotient is calculated by seven primary subtests similarities, vocabulary, block design, matrix reasoning, figure weights, digit span, and coding. 


\section{Method}

\subsection{Population and procedure}

Access to colleges and primary schools in the city was carried by authorizations of the regional academies of education and training in Safi also the directors of the institutions. We administered all subtests of test by respecting the age order, for each student participates from the Safi city. The participants are 101 students (59 girls and 42 boys) age 12 years and 13 years and some months (mean age 12 years 8 months 27 days standard deviation 00 years 4 months 17 days). We also carried a parental questionnaire about the household income and educational level of parents, grandparents, uncles, and aunts. The samples are students from the middle and primary schools in Safi. We chose students following the proposal of the teachers by agreement of the parents. The administration is according to their availability during the vacations school hours, on the premises of the colleges and schools. The all students are from the urban environment despite the existence of internal Students in the College we did not study its students because of the impact of the urban environment on intellectual development respecting the age order of WISC V.

\subsection{Instrument}

We worked by the French version of the Wechsler intelligence scale for children WISC V, this intelligence test is used to determine whether a cognitive impairment underlies an academic difficulty, or to predict a child's ability to benefit from a special education placement. To achieve these goals, the current French version of WISC (WISC-V) distinguishes five indexes, the verbal comprehension index VCI [similarities, vocabulary, information, comprehension], visuospatial index VSI [block design, visual puzzles], fluid reasoning index FRI [matrix reasoning, figure weights, arithmetic], working memory index WMI [digit span, picture span, letter-number sequencing], and processing speed index psi [coding, symbols search, cancellation]. in addition to full scale intelligence quotient FSIQ, it's calculated by seven subtests on five areas the subtests are similarities, vocabulary, block design, matrix reasoning, figure weights, digit span and coding. also according to this test five additional indexes calculate quantitative reasoning (figure weights and arithmetic), auditory working memory (digit span and letter-number sequencing), non-verbal (block design, visual puzzles, matrix reasoning, figure weights, picture span and coding), general ability (similarities, vocabulary, block design, matrix reasoning and figure weights) and cognitive abilities (digit span, picture span, coding and symbol search). Thus, the calculation of three additional indexes from the complementary subtests the naming speed index, the symbol association index (or associative memory) and the information storage and retrieval index. The WISC $\mathrm{V}$ test for children aged 6 to 16 years and 9 months. Usually lasts between 60 and 80 minutes and produces a fairly typical result.

\section{Result}

The table 1 show the mean values and standard deviations of the five indexes and the full scale intelligence quotient FSIQ of the participants in this test. We find that the two indexes: visuospatial index VSI and fluid reasoning index FRI are slightly lower than the theoretical mean 100, the two indexes: working memory index WMI and processing speed index PSI are above the theoretical average. Only, the verbal comprehension index VCI is slightly above average.

Figure 1. Indexes Scores of urban pupils.

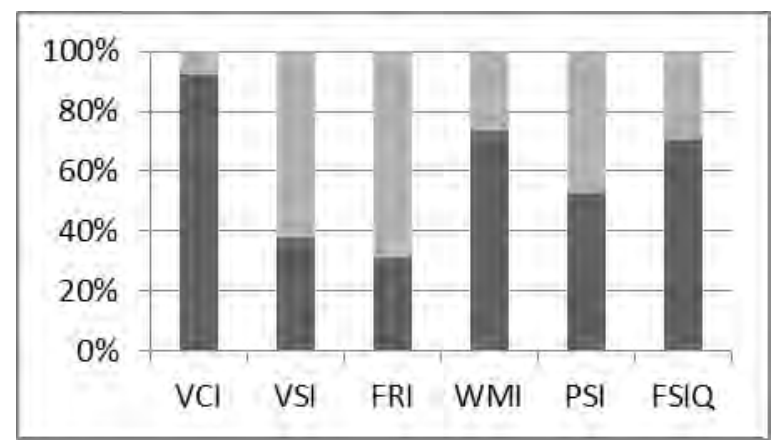


The black in the figure 1 present the percentage of students with index scores greater than or equal to 100 and in grey show the percentage of students with index scores less than 100 .

The attracted particular attention the difference between the VCI and the index closest to the WMI is 20 points, or one standard deviation higher. The standard deviation of the two indexes VCI and WMI is different by 6 points. The WMI is different from PSI by 6 points or half a standard deviation. The difference between the PSI and the two indexes the VSI and the FRI is 7 points. Note that the figure combines the percentages of children scoring high and low on the indexes and the FSIQ.

Table 1. $M=$ Mean, $S D=$ Standard Deviation, $V C I=$ Verbal Comprehension Index, VSI = Visuospatial index, $F R I=$ Fluid Reasoning Index, WMI= Working Memory Index, PSI= Processing Speed Index, FSIQ=Full Scale Intelligence Quotient.

\begin{tabular}{|c|c|c|c|c|c|c|}
\hline & VCI & VSI & FRI & WMI & PSI & FSIQ \\
\hline M & 127,54 & $\mathbf{9 4 , 9}$ & $\mathbf{9 3 , 6 7}$ & $\mathbf{1 0 7 , 4}$ & $\mathbf{1 0 1 , 7 7}$ & $\mathbf{1 0 7 , 8 7}$ \\
\hline SD & 19,31 & $\mathbf{1 0 , 8 5}$ & $\mathbf{1 0 , 9 4}$ & $\mathbf{1 3 , 9 6}$ & $\mathbf{1 5 , 2 7}$ & $\mathbf{1 3 , 6 7}$ \\
\hline
\end{tabular}

For the three indexes VCI, WMI, PSI and FSIQ a large percentage of children scored above the theoretical average 100, which is shown in the figure, whereas the percentage of children scored above the theoretical average 100 in the two indexes VSI and FRI does not exceed $40 \%$. Table 2 shows the correlation matrix between the five WISC V indexes administered in the samples.

Table 2. Matrix of correlations between the five WISC V indexes for the children.

\begin{tabular}{|c|c|c|c|c|}
\hline & VCI & VSI & FRI & WMI \\
\hline VSI & $\mathbf{0 , 4 7}$ & & & \\
\hline FRI & $\mathbf{0 , 4 9}$ & $\mathbf{0 , 5 9}$ & & \\
\hline WMI & $\mathbf{0 , 3 8}$ & $\mathbf{0 , 3 6}$ & $\mathbf{0 , 4 2}$ & \\
\hline PSI & $\mathbf{0 , 3 8}$ & $\mathbf{0 , 3 3}$ & $\mathbf{0 , 3 7}$ & $\mathbf{0 , 4 3}$ \\
\hline
\end{tabular}

It can be seen that the correlation between the two indexes the FRI and the VSI with a low mean is a strong positive relationship. The other correlations between all five indexes are between strong positive relationship and moderate positive relationship.

The results of the scores of the WISC V psychometric test for Moroccan students are given a Cronbach alpha coefficient of 0.87 in the urban environment. Also, the correlation between the average of the class scores of the two semesters and the FSIQ for the participating students is 0.56 . Not to mention that girls scored 108.64 in the FSIQ higher than the 106.79 score obtained by boys. The confirmatory factor analysis to one-factor yielded satisfactory adjustment statistics; RMSEA $=.047$, CFI $=.965$ and $\mathrm{TLI}=.954$. Based on the results of the exploratory factor analysis, a one-factor model was tested with the sample. The two- and three-factor models did not yield higher saturation coefficients and communities than the one-factor model.

\section{Discussion}

The administration of WISC $\mathrm{V}$ for Moroccan schoolchildren in urban areas received a very significant Cronbach's coefficient on the administration of the test between students, so the correlation between the full scale intelligence quotient scores and the school year average scores gave a strong positive correlation. Girls scored higher on the FSIQ, which is consistent with the difference in intelligence of gender despite the number of girls participating in this study being more than half of all samples. The level of education attained was also found to be an important social factor in IQ. Gender and education are known contextual variables that predict performance on IQ tests. Test duration was an important factor in our subjects, with several students being treated in two sessions.

The average value of the full scale intelligence quotient is higher than the theoretical average. Clinicians have often argued that intelligence quotient can misrepresent an individual's level of cognitive functioning when differences between component scores are extreme (Kamphaus, 2001; Kaufman, 1994; Prifitera et al., 1998).

For the results of our studies that show a high mean of the verbal comprehension index to the theoretical mean of the samples even for girls and boys, the standard deviation of this index is also high compared to other standard deviations. This index mobilizes the knowledge acquired over the course of 
life and is the best indicator of crystallized intelligence, which implies the academic performance of the participants. It can be seen that several students reach 155 in the results of this test, which is identical with the results obtained by high potential students. Most of the parents are educated without a high level of education, so all the samples are educated in the pre-school period.

The working memory index and the processing speed index are higher than the theoretical mean hundred and their standard deviation of both indexes is close to each other. The working memory index is responsible for the learning, which is well shown for the results of the children participating in this study, as well as the percentage of students with above-average scores. And concerning the processing speed index, which means the speed of information processing, the results are satisfactory for this sample of students because a little more than half of them are above one hundred.

The visuospatial index which calculates visual intelligence is below average, for this index the students with low scores could predict difficulties in mathematics at school which shows their academic performance in mathematics. The fluid reasoning index is lower than the mean and the standard deviation is close to the visuospatial index. It is a specific form of fluid intelligence (Gf) which represents the ability to solve new or abstract problems and which also involves some quantitative knowledge.

The mean values of the full scale intelligence quotient for each level for the participants show higher values for the verbal comprehension index VCI compared to the other indexes Gregoire (2009) has also shown the influence that these variables can have on IQ scores and particularly on the verbal comprehension index VCI. On the other hand, proponents of a multifactorial intelligence model assume that there is a variety of cognitive abilities and that useful intelligence tests should measure a variety of abilities rather than a single IQ (e.g., Guilford, 1967; Horn \& Cattell, 1967; Thurstone, 1938).

\section{Conclusion}

Administration of the Wechsler Child and Adolescent Intelligence Scale for the full scale intelligence quotient in urban areas revealed the cognitive profiles of the participants in this study: we found low income per dominant household among the schoolchildren and that their parents and grandparents were illiterate. The full scale intelligence quotient of the participants in this test is on average above the theoretical average. The two indexes visuospatial index IVS and the fluid reasoning index FRI are found to be slightly below the theoretical average and the two indexes working memory WMI and processing speed PSI are above the theoretical average. Only the verbal comprehension index VCI is slightly above average. The results are very significant for the cognitive state need to develop in low scoring students also to look for other types of intelligence to process and to direct students towards specific training.

\section{References}

Alfieri, L., Brooks, P. J., Aldrich, N. J., \& Tenenbaum, H. R. (2011). Does discovery-based instruction enhance learning? Journal of Educational Psychology, 103(1), 1-18.

Carroll, J. B. (1993). Human cognitive abilities: A survey of factor analytic studies. New York: Cambridge University Press.

Cattell, R. B. (1941). General psychology. Oxford, England: Sci-Art.

Deary, I. J., Strand, S., Smith, P., \& Fernandes, C. (2007). Intelligence and educational achievement. Intelligence, 35, 13-21.

Glutting, J. J., McDermott, P. A., Konold, T. R., Snelbaker, A. J., \& Watkins, M. W. (1998). More ups and downs of subtest analysis: criterion validity of the DAS with an unselected cohort. School Psychology Review, 27, 599-612.

Glutting, J. J., Youngstrom, E. A., Ward, T., Ward, S., \& Hale, R. L. (1997). Incremental validity of WISC-III factor scores in predicting achievement: what do they tell us? Psychological Assessment, 9, 295-301.

Guilford, J. P. (1967). The nature of human intelligence. New York: McGraw-Hill.

Horn, J. L. (1965). A rationale and test for the number of factors in factor analysis. Psychometrika, 30, $179-185$. http://dx.doi.org/10.1007/BF02289447

Horn, J. L., \& Cattell, R. B. (1967). Age differences in fluid and crystallized intelligence. Acta Psychologica, 26, 107-129.

Kamphaus, R. W. (2001). Clinical assessment of child and adolescent intelligence (2nd ed.). Needham Heights, MA: Allyn and Bacon.

Kaufman, A. S. (1994). Intelligent testing with the WISC-III. New York: Wiley. 
McDermott, P. A., Fantuzzo, J. W., \& Glutting, J. J. (1990). Just say no to subtest analysis: a critique on Wechsler theory and practice. Journal of Psychoeducational Assessment, 8, 290-302.

Prifitera, A., Weiss, L. G., \& Saklofske, D. H. (1998). The WISC-III in context. In A. Prifitera, \& D. H.

Rohde, T. E., \& Thompson, L. A. (2007). Predicting academic achievement with cognitive ability. Intelligence, 35(1), 83-92. https://doi.org/10.1016/j.intell.2006.05.004.

Roth, B., Becker, N., Romeyke, S., Schafer, S., Domnick, F., \& Spinath, F. M. (2015). Intelligence and school grades: A meta-analysis. Intelligence, 53, 118-137. https://doi.org/10.1016/j.intell.2015.09.002.

Spearman, C. (1904). "General Intelligence", objectively determined and measured. The American Journal of Psychology, 15, 201-292.

Thurstone, L.L. (1938). Primary mental abilities. : University of Chicago Press: Chicago.

Watkins, M. W. (2000). Cognitive profile analysis: a shared professional myth. School Psychology Quarterly, 15, 465-479.

Wechsler, D. (1939). Wechsler-Bellevue Intelligence Scale. New York: The Psychology Corporation.

Wechsler, D. (2016a). WISC-V. Echelle d'intelligence de Wechsler pour enfants-5e édition. Paris, France: Pearson France-ECPA.

Wechsler, D. (2016b). WISC-V. Echelle d'intelligence de Wechsler pour enfants-5e édition, manual d'interprétation. Paris, France: Pearson France-ECPA. 